\title{
Rorschach Sistema Compreensivo na avaliação de psicoterapia psicodinâmica
}

\author{
Latife Yarigi - Universidade Federal de São Paulo, São Paulo, Brasil \\ Terezinha de Carvalho Amaro - Universidade Federal de São Paulo, São Paulo, Brasil \\ Maria Luiza de Mattos Fiore - Universidade Federal de São Paulo, São Paulo, Brasil \\ Norma Lottenberg Semer - Universidade Federal de São Paulo, São Paulo, Brasil
}

\begin{abstract}
Resumo
A proposta foi avaliar mudanças decorrentes de psicoterapia psicanalítica em pessoas assistidas em um serviço universitário. Assim, 68 pessoas adultas, de ambos os sexos, foram avaliadas antes e depois de um ano de psicoterapia. Os protocolos de Rorschach Sistema Compreensivo foram analisados de acordo com seu grau de complexidade, metade em "menor" $[R \downarrow+F \uparrow]$ e metade em "maior" $[R \uparrow+F \downarrow]$. Diferenças estatisticamente significantes foram encontradas com (a) aumento no grupo de "menor" complexidade e diminuição no grupo de "maior" complexidade em: R; EA; es; Sum Y; Sum Shd; FC+CF+C;CF+C; $M$; ativo; FD; $H+(H)+H d+(H d)$; H puro, DEPI, (b) diminuição no "menor" e aumento no "maior" em Lambda e F puro. Observouse confluência dos resultados, com o grupo de "maior" complexidade com menos turbulência em suas emoções e o de "menor" complexidade expressando abertura às experiências, mesmo se desconfortáveis. A terapia mostrou trazer benefícios a essas pessoas.

Palavras-chave: Rorschach Sistema Compreensivo, Avaliação de resultado de intervenções terapêuticas, psicoterapia Psicanalítica.
\end{abstract}

Rorschach Comprehensive System on the evaluation of psychodynamic psychotherapy

\begin{abstract}
The proposal aimed to evaluate changes due to psychoanalytic psychotherapy on individuals assisted in a university clinic. Thus, 68 adult subjects, both genders, were evaluated before and after one year of psychotherapy. The Rorschach Comprehensive System protocols were analyzed according to their level of complexity, half on "low" $[R \downarrow+F \uparrow]$ and half on "high" $[R \uparrow+F \downarrow]$. Significant statistical differences were found for (a) increase in "low" complexity group and decrease in 'high' complexity group on: R; EA; es; SumY; SumShd; FC+CF+C; $C F+C ; M$; active; FD; $H+(H)+H d+(H d)$; H pure, DEPI, (b) decrease in "low" and increase in "high" on Lambda and F pure. A confluence of these results was observed; the "high" complexity group showing less turbulence of their emotions, and the "low" complexity group displaying openness to experiences, even if of a more discomforting kind. The therapy proved to be beneficial to the individuals.

Keywords: Rorschach Comprehensive System, Evaluation of results of therapeutic interventions, Psychoanalityc psycotherapy.
\end{abstract}

A proposta consistiu em avaliar as mudanças advindas de tratamento psicoterápico de pessoas atendidas no Centro Clínico de Pesquisa em Psicoterapia do Departamento de Psiquiatria da Universidade Federal de São Paulo. Por se tratar de uma instituição de ensino público, são atendimentos supervisionados realizados em sua "maioria" por profissionais em treinamento, especializandos do Programa Psicologia da Saúde e médicos do Programa de Residência em Psiquiatria. A abordagem adotada pela equipe de supervisores é a psicodinâmica, com atendimentos semanais, sempre em uma mesma sala. As avaliações ocorrem antes do início da psicoterapia e em acompanhamentos anuais até a finalização do tratamento.

As pessoas encaminhadas para psicoterapia são provenientes dos diferentes ambulatórios do Hospital São Paulo, hospital-escola da universidade, por outros serviços públicos ou por procura espontânea. Inicialmente, são submetidas à entrevista de triagem, com o intuito de confirmar ou não a

1 Endereço para correspondência:

Rua Manoel Guedes, 385 - ap. 11 - 04536-070 - São Paulo-SP indicação de psicoterapia. Em seguida, são informadas sobre o estudo que vincula $\mathrm{O}$ atendimento psicoterápico à participação em protocolos de pesquisa. Dessa forma, após o consentimento livre e esclarecido assinado, as avaliações são iniciadas, com a aplicação da Entrevista Clínica Estruturada para o DSM-IV, SCID-I e SCID-II (Del-Ben \& cols., 2001) por psiquiatras treinados. Em continuidade, são realizadas as avaliações psicológicas, dentre elas a aplicação do Método de Rorschach, Sistema Compreensivo (Exner, 2003), instrumento privilegiado de investigação. As reavaliações ocorrem a cada 12 meses da data de início.

\section{Estudo}

O desenho do presente estudo tem por base o método clínico-qualitativo (Morse \& Field, 1995) dada a intenção de se buscar explicação e significado atribuídos ao fenômeno estudado (Turato, 2003). Trata-se de uma série de estudos de caso clínicos de indivíduos, na forma de teste-reteste, acompanhados em ambulatório psicoterápico em uma instituição universitária gratuita. A amostragem é intencional estratificada dada a seleção intencional e categórica dos 
elementos selecionados para a amostra e a possibilidade de comparar por triangulação os resultados obtidos pelo Rorschach com os diagnósticos feitos pelas entrevistas clínicas estruturadas (Patton, 2002).

Os critérios de inclusão do participante são: motivação, interesse e disponibilidade efetiva para frequentar as sessões. Os critérios de exclusão são: pessoas com diagnóstico de esquizofrenia, demência e retardo mental, de acordo com a Entrevista Clínica Estruturada.

\section{Resultados}

Foram avaliados 68 indivíduos antes e após 12 meses de psicoterapia, 48 mulheres e 20 homens; idade variando de 19 a 72 anos (média de 39 anos, DP= $11,4) ; 33(48 \%)$ solteiros e 21 (31\%) casados; 32 $(48,5 \%)$ com 12 ou mais anos de escolaridade e 22 $(32,4 \%)$ com 9 a 11 anos de escolaridade; 35 (51,5\%) pertencentes à classe média baixa, $17(25,0 \%)$ à classe média e 16 (23,5\%) à classe baixa; 24 (35,5\%) desempregados, 11 (16,2\%) inativos, 18 (26,5 ativos), $10(14,7 \%)$ donas de casa e $5(7,3 \%)$ estudantes. Assim, a maioria é composta por mulheres, estado civil solteiro, escolaridade ensino superior, nível sócioeconômico médio-baixo e sem trabalho.

A avaliação diagnóstica foi feita por meio da aplicação de entrevistas clínicas estruturadas para diagnóstico, SCID I e II, de acordo com a quarta edição da DSM, Diagnostic and Statistical Manual of Mental Disorders (American Psychiatric Association, 1994). Esta classificação é baseada na descrição fenomenológica de um conjunto de manifestações dos transtornos mentais. Seu sistema de avaliação inclui cinco eixos: I - síndromes clínicas, II - transtornos de desenvolvimento e personalidade, III - transtornos e condições físicas, IV - gravidade de estressores psicossociais, e V - avaliação global do funcionamento. As Entrevistas Clínicas Estruturadas foram desenvolvidas para garantir a validade e a confiabilidade nos diagnósticos psiquiátricos do eixo I (SCID-I) e do eixo II (SCID-II).

Dos 68 participantes, 43 (63,2\%) preenchem critério para transtorno depressivo maior no Eixo I e
$18(26,4 \%)$ preencheram critério para transtorno de personalidade evitante, $9(13,2 \%)$ para transtorno de personalidade borderline no Eixo II. Seis $(8,8 \%)$ participantes não preenchem critérios para o Eixo I e $18(26,4 \%)$ não preenchem critérios para o Eixo II.

Estudo comparativo das variáveis do Rorschach entre dois momentos de avaliação, antes e após um ano de psicoterapia

As variáveis do Rorschach selecionadas para análise foram pautadas no texto de Weiner (2000) sobre interpretação do Método de Rorschach no Sistema Compreensivo. Como são 214 variáveis, elegeram-se as mais significativas no contexto do processo psicoterápico.

Optou-se por seguir a proposta de Dean, Viglioni, Perry e Meyer (2007) e considerar os protocolos de acordo com sua complexidade. Os de "maior" complexidade seriam aqueles com "maior" número de respostas e "menor" número de respostas de forma $(\mathrm{R} \uparrow$ e $\mathrm{F} \downarrow)$, e os de "menor" complexidade seriam aqueles com "menor" número de respostas e "maior" número de respostas de forma ( $\mathrm{R} \downarrow$ e $\mathrm{F} \uparrow$ ).

A análise estatística considerou protocolos de "maior" e "menor" complexidade e o tempo de avaliação, antes e depois de um ano de psicoterapia, e teve como procedimento específico a análise de variância com medidas repetidas (Tabachinick \& Fidell, 1996). Por meio dessa análise, foi possível verificar se o perfil de médias de um conjunto de medidas (parte intrassujeito do delineamento) foi diferente para grupos distintos (parte entre-sujeitos do delineamento) (Primi \& cols., 2000). Foram empregadas ANOVA $2 \mathrm{x}$ 2 , uma vez que os participantes haviam respondido duas vezes o instrumento.

Dos 68 sujeitos avaliados, 35 compuseram o grupo de "maior" complexidade, indicado como 1,00, e 33 o grupo de "menor" complexidade, indicado como ,00. Na Tabela 1, os resultados das variáveis que foram significativas nas comparações entre os dois momentos, levando-se em conta complexidade*tempo, e para "maior" complexidade 1,00, e para "menor" complexidade ,00, e em seguida suas representações na forma de gráficos. 
Tabela 1 - Diferenças entre as médias das variáveis do Rorschach entre a $1^{\mathrm{a}}$ e $2^{\mathrm{a}}$ aplicação, de acordo com a complexidade, maior ou ,00 e menor ou 1,00 (continua)

\begin{tabular}{|c|c|c|c|c|c|c|c|}
\hline Variáveis & Complexidade & Média & $\mathrm{DP}$ & $\mathrm{N}$ & $\mathrm{F}$ & Sig. & $\mathrm{Eta}^{2}$ \\
\hline & , 00 & 18,88 & 4,121 & 33 & \multirow{6}{*}{6,322} & \multirow{6}{*}{0,014} & \multirow{6}{*}{0,087} \\
\hline \multirow[t]{3}{*}{ R1 } & 1,00 & 28,03 & 9,627 & 35 & & & \\
\hline & Total & 23,59 & 8,739 & 68 & & & \\
\hline &, 00 & 20,12 & 6,318 & 33 & & & \\
\hline \multirow[t]{2}{*}{ R 2} & 1,00 & 24,29 & 8,525 & 35 & & & \\
\hline & Total & 22,26 & 7,768 & 68 & & & \\
\hline \multirow[t]{3}{*}{ Lambda.1 } &, 00 & 1,2852 & ,56682 & 33 & & & \\
\hline & 1,00 &, 5640 & 39903 & 35 & & & \\
\hline & Total & ,9140 & 60506 & 68 & & & 050 \\
\hline \multirow[t]{3}{*}{ Lambda.2 } &, 00 & 1,0324 & 80061 & 33 & 4,114 & ,047 & (059 \\
\hline & 1,00 & ,6634 & 49197 & 35 & & & \\
\hline & Total & ,8425 & 68079 & 68 & & & \\
\hline \multirow[t]{3}{*}{$\%$ Fpuro 1} &, 00 & ,5376 & 10574 & 33 & & & \\
\hline & 1,00 & ,3314 & , 12612 & 35 & & & \\
\hline & Total & ,4315 & , 15552 & 68 & & & \\
\hline \multirow[t]{3}{*}{$\%$ Fpuro 2} &, 00 & ,4464 & 17841 & 33 & 7,298 & - 2009 & 100 \\
\hline & 1,00 & 3580 & 15031 & 35 & & & \\
\hline & Total & ,4009 & ,16925 & 68 & & & \\
\hline \multirow[t]{3}{*}{$E A .1$} &, 00 & 3,6818 & 2,03031 & 33 & & & \\
\hline & 1,00 & 8,4286 & 3,88652 & 35 & & & \\
\hline & Total & 6,1250 & 3,91741 & 68 & & & \\
\hline \multirow[t]{3}{*}{ EA.2 } &, 00 & 4,9545 & 3,29147 & 33 & 11,064 & - , & , 144 \\
\hline & 1,00 & 6,8857 & 3,94090 & 35 & & & \\
\hline & Total & 5,9485 & 3,74180 & 68 & & & \\
\hline \multirow[t]{3}{*}{ Es.1 } &, 00 & 5,3939 & 2,81668 & 33 & & & \\
\hline & 1,00 & 13,6000 & 6,18442 & 35 & & & \\
\hline & Total & 9,6176 & 6,34583 & 68 & & & \\
\hline \multirow[t]{3}{*}{ Es.2 } &, 00 & 7,5152 & 4,41674 & 33 & 7,090 & , & , 097 \\
\hline & 1,00 & 11,6286 & 6,64692 & 35 & & & \\
\hline & Total & 9,6324 & 6,00225 & 68 & & & \\
\hline \multirow[t]{3}{*}{ SumY.1 } &, 00 &, 8485 & 1,03444 & 33 & & & \\
\hline & 1,00 & 3,2286 & 3,37888 & 35 & & & \\
\hline & Total & 2,0735 & 2,78223 & 68 & & & \\
\hline \multirow[t]{3}{*}{ SumY.2 } &, 00 & 1,9697 & 2,37809 & 33 & 10,351 & , &, 136 \\
\hline & 1,00 & 2,2857 & 2,56184 & 35 & & & \\
\hline & Total & 2,1324 & 2,46107 & 68 & & & \\
\hline \multirow[t]{3}{*}{ SumShd.1 } &, 00 & 2,3030 & 1,92816 & 33 & & & \\
\hline & 1,00 & 6,8571 & 4,94168 & 35 & & & \\
\hline & Total & 4,6471 & 4,40747 & 68 & 5247 & 025 & 074 \\
\hline \multirow[t]{3}{*}{ SumShd.2 } &, 00 & 3,9697 & 3,56620 & 33 & $5,24 /$ &, 025 &, $0 / 4$ \\
\hline & 1,00 & 6,0286 & 4,94966 & 35 & & & \\
\hline & Total & 5,0294 & 4,42507 & 68 & & & \\
\hline \multirow[t]{3}{*}{$F C+C F+C .1$} &, 00 & 2,3636 & 1,85098 & 33 & & & \\
\hline & 1,00 & 5,0857 & 2,98399 & 35 & & & \\
\hline & Total & 3,7647 & 2,83432 & 68 & & & \\
\hline \multirow[t]{3}{*}{$F C+C F+C .2$} &, 00 & 3,1515 & 2,50151 & 33 & 4,297 & , 042 & ,061 \\
\hline & 1,00 & 4,5143 & 2,97412 & 35 & & & \\
\hline & Total & 3,8529 & 2,81926 & 68 & & & \\
\hline$C F+C .1$ &, 00 & 1,3030 & 1,38033 & 33 & & & \\
\hline & 1,00 & 2,4000 & 1,95839 & 35 & &, 006 & 109 \\
\hline & Total & 1,8676 & 1,77801 & 68 & 8,094 & & \\
\hline
\end{tabular}


Tabela 1 - Diferenças entre as médias das variáveis do Rorschach entre a $1^{\mathrm{a}}$ e $2^{\mathrm{a}}$ aplicação, de acordo com a complexidade, maior ou ,00 e menor ou 1,00 (conclusão)

\begin{tabular}{|c|c|c|c|c|c|c|c|}
\hline Variáveis & Complexidade & Média & DP & $\mathbf{N}$ & $\mathrm{F}$ & Sig, & $\mathrm{Eta}^{2}$ \\
\hline \multirow[t]{3}{*}{$C F+C .2$} & , 00 & 1,7879 & 1,84996 & 33 & & & \\
\hline & 1,00 & 1,7143 & 1,42605 & 35 & & & \\
\hline & Total & 1,7500 & 1,63337 & 68 & & & \\
\hline \multirow[t]{3}{*}{ M.1 } &, 00 & 1,6970 & 1,40278 & 33 & & & \\
\hline & 1,00 & 4,4857 & 2,99383 & 35 & 7,144 & ,009 & ,098 \\
\hline & Total & 3,1324 & 2,73127 & 68 & & & \\
\hline \multirow[t]{3}{*}{ M.2 } &, 00 & 2,2121 & 2,54654 & 33 & & & \\
\hline & 1,00 & 3,4857 & 3,07142 & 35 & & & \\
\hline & Total & 2,8676 & 2,88022 & 68 & & & \\
\hline \multirow[t]{3}{*}{ Ativo.1 } &, 00 & 2,2121 & 2,14706 & 33 & & & \\
\hline & 1,00 & 6,2857 & 3,28608 & 35 & & & \\
\hline & Total & 4,3088 & 3,44794 & 68 & & & \\
\hline \multirow[t]{3}{*}{ Ativo.2 } & ,00 & 2,9394 & 2,49924 & 33 & 11,399 & 001 & 147 \\
\hline & 1,00 & 4,4857 & 3,16573 & 35 & & & \\
\hline & Total & 3,7353 & 2,94536 & 68 & & & \\
\hline \multirow[t]{3}{*}{ FD.1 } &, 00 & ,4545 & 61699 & 33 & & & \\
\hline & 1,00 & 1,6571 & 1,71401 & 35 & & & \\
\hline & Total & 1,0735 & 1,42804 & 68 & 6800 & 011 & 005 \\
\hline \multirow[t]{3}{*}{ FD. 2} &, 00 & ,8788 & 1,51570 & 33 & 0,899 & (011, & , \\
\hline & 1,00 & 1,0571 & 1,25892 & 35 & & & \\
\hline & Total & ,9706 & 1,38187 & 68 & & & \\
\hline \multirow[t]{3}{*}{$H+(H)+H d+(H d) .1$} &, 00 & 3,7576 & 2,04680 & 33 & & & \\
\hline & 1,00 & 8,6857 & 6,45241 & 35 & & & \\
\hline & Total & 6,2941 & 5,41159 & 68 & 7635 & 007 & 104 \\
\hline \multirow{3}{*}{$H+(H)+H d+(H d) \cdot 2$} &, 00 & 4,2424 & 3,76688 & 33 & , ,,035 & 1001, & 104 \\
\hline & 1,00 & 7,0000 & 6,32456 & 35 & & & \\
\hline & Total & 5,6618 & 5,38547 & 68 & & & \\
\hline \multirow[t]{3}{*}{ NPH.1 } &, 00 & 2,2424 & 1,69614 & 33 & & & \\
\hline & 1,00 & 5,5429 & 4,44802 & 35 & & & \\
\hline & Total & 3,9412 & 3,76505 & 68 & 5880 & & \\
\hline \multirow[t]{3}{*}{ NPH.2 } &, 00 & 2,7576 & 2,87261 & 33 & 5,869 & ,018 &, 082 \\
\hline & 1,00 & 4,5143 & 4,42168 & 35 & & & \\
\hline & Total & 3,6618 & 3,82689 & 68 & & & \\
\hline \multirow[t]{3}{*}{ DEPI pos.1 } &, 00 & ,2121 & ,41515 & 33 & & & \\
\hline & 1,00 & ,4571 &, 50543 & 35 & & & \\
\hline & Total & ,3382 & ,47663 & 68 & 4453 & 030 & 063 \\
\hline \multirow[t]{3}{*}{ DEPI pos. 2} &, 00 & ,3636 & ,48850 & 33 & 4,435 & צ'0נ, & נינ, \\
\hline & 1,00 & ,3429 & ,48159 & 35 & & & \\
\hline & Total & ,3529 & ,48144 & 68 & & & \\
\hline
\end{tabular}

1. Número de respostas $-R$ - ou produção associativa.

$\mathrm{Na}$ comparação entre os dois momentos, observa-se aumento do número de respostas nos protocolos de "menor" complexidade e diminuição naqueles de "maior" complexidade, em direção aos valores médios. Nos gráficos visualiza-se tendência à aproximação dos dois níveis de complexidade. Assim, nas pessoas com atitudes mais superficiais ou econômicas, a psicoterapia estimulou a produção de associações em que ocorreu maior diversidade de aspectos $\mathrm{da}$ personalidade que antes não se expressaram. Já nas pessoas com maior envolvimento da subjetividade na tarefa, ocorreu certa restrição em direção a um equilíbrio no foco de atenção. 
Estimated Marginal Means of MEASURE_1

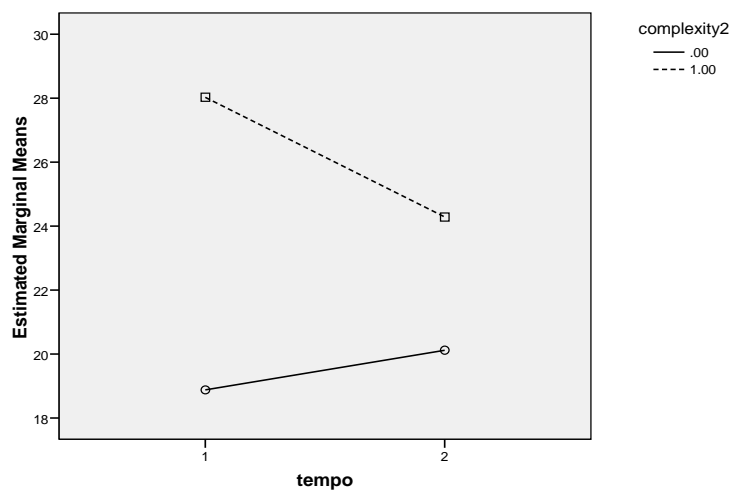

Legenda: $.00=$ menor complexidade; $1.00=$ maior complexidade; $1=1^{\mathrm{a}}$ aplicação; $2=2^{\mathrm{a}}$ aplicação

Figura 1 - Resultados da variável $R$ nos grupos de menor e maior complexidade na $1^{a}$ e $2^{a}$ aplicações

2. Lambda - razão que compara a frequência de respostas de forma pura em relação às demais respostas do protocolo [F puras/R-F]. Relaciona-se à questão da economia do uso dos recursos internos. $\mathrm{Da}$ mesma forma que ocorre com número de resposta, evidencia-se confluência entre dados dos protocolos de "maior" e "menor" complexidade. Assim, as pessoas mais fechadas à experiência passaram a se expor mais e as mais abertas se recolhem em direção a uma moderação. Desta maneira, a psicoterapia vai promovendo um equilíbrio entre o foco de atenção, ampliando-o no grupo mais superficial e restringindo-o no grupo de "maior" complexidade. Weiner (2000) comenta que pessoas que mantêm um foco de atenção equilibrado tendem a se dar conta do que ocorre interna e externamente, a tolerar ambiguidades e incertezas, a se interessar mais e a ser capazes de lidar com as situações de uma maneira flexível.

Estimated Marginal Means of MEASURE_1

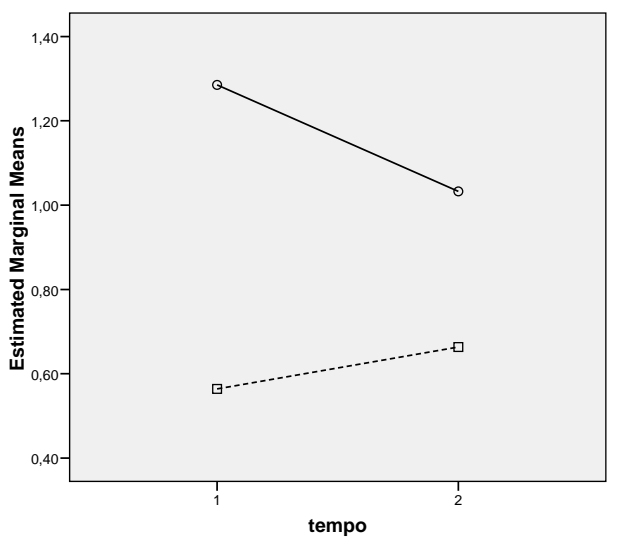

complexity2 $-.00$

Legenda: $.00=$ menor complexidade; $1.00=$ maior complexidade; $1=1^{\mathrm{a}}$ aplicação; $2=2^{\mathrm{a}}$ aplicação

Figura 2 - Resultados da variável Lambda nos grupos de menor e maior complexidade na $1^{\mathrm{a}}$ e $2^{\mathrm{a}}$ aplicações

3. Respostas $F$ puro - refere-se à atitude mais formal, superficial e distante, podendo significar falta de recursos pessoais ou atitude de defesa em se expor. Corroborando os dados acima, o grupo de "menor" complexidade diminui de modo marcado o valor da variável Forma, enquanto o grupo de "maior" complexidade apresenta um aumento moderado. Como é possível constatar no gráfico, as tendências se aproximam; assim as pessoas mais contidas vão podendo expor mais os aspectos de sua personalidade, afetivos, emocionais e ideativos, e as mais espontâneas mostram-se mais comedidas. 
Estimated Marginal Means of MEASURE_1

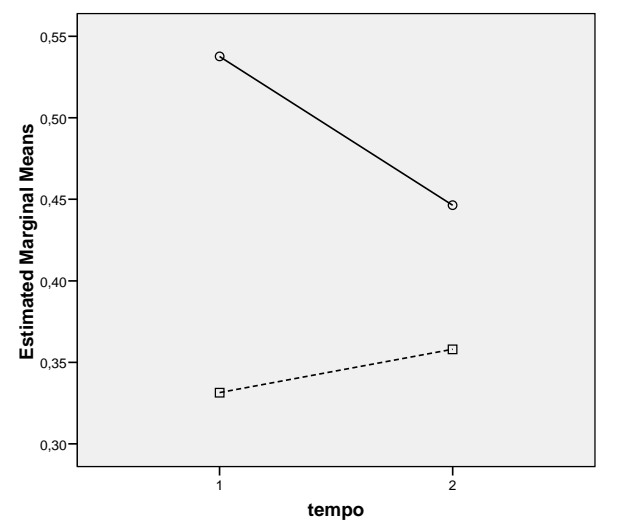

Legenda: $.00=$ menor complexidade; $1.00=$ maior complexidade; $1=1^{\mathrm{a}}$ aplicação; $2=2^{\mathrm{a}}$ aplicação

Figura 3 - Resultados da variável $\%$ F puro nos grupos de menor e maior complexidade na $1^{\mathrm{a}}$ e $2^{\mathrm{a}}$ aplicações

4. Experiência efetiva - EA - variável associada aos recursos disponíveis adequados para atender às demandas do cotidiano, cuja fórmula é obtida somando-se os dois lados de $E B[M+W S u m C]$. Da mesma forma que as variáveis anteriores, os protocolos de "menor" complexidade apresentaram elevação dessa variável e os de "maior" complexidade, uma diminuição na comparação entre os dois momentos, ambos confluindo para valores esperados. Assim, a terapia promoveu maior abertura para a experiência, maior participação dos aspectos ideativos e afetivos nas pessoas que inicialmente expressaram poucos recursos internos e possibilitou maior aproveitamento do potencial interno, ideativo e afetivo, naquelas pessoas que manifestaram mais recursos internos.

\section{Estimated Marginal Means of MEASURE_1}

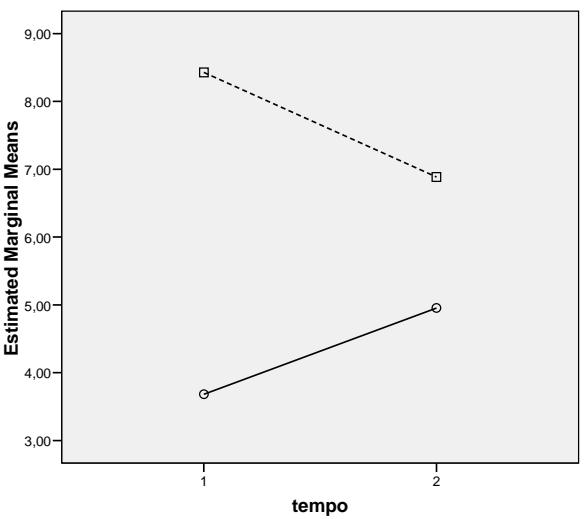

Legenda: $.00=$ menor complexidade; $1.00=$ maior complexidade; $1=1^{\mathrm{a}}$ aplicação; $2=2^{\mathrm{a}}$ aplicação

Figura 4 - Resultados da variável $E A$ nos grupos de menor e maior complexidade na $1^{\mathrm{a}}$ e $2^{\mathrm{a}}$ aplicações

5. Estimulação sentida - es - relaciona-se com as demandas atuais dos estímulos, cuja formula é obtida somando-se os dois lados de $e b\left[F M+m+\operatorname{Sum} C^{\prime}+\right.$ $\operatorname{Sum} T+\operatorname{Sum} Y+\operatorname{Sum}[$.

Novamente, na comparação entre os dois momentos, os protocolos de "menor" complexidade apresentaram elevação e os de "maior" complexidade, apesar de uma queda, se mantêm acima do esperado. Assim, as pessoas mais contidas ou superficiais passaram a expressar mais suas tendências ideativas, mesmo as imaturas, e as emoções, mesmo as desconfortáveis, enquanto as mais abertas mantêm, de modo menos intenso, as manifestações ideativas e emocionais. É esperado que a psicoterapia mobilize tais expressões, e nas pessoas com mais recursos observou-se diminuição da participação desses aspectos após um ano de terapia. Assim, as pessoas se 
permitem expressar seu desconforto emocional, suas angústias, ansiedades e medos.

Estimated Marginal Means of MEASURE_1

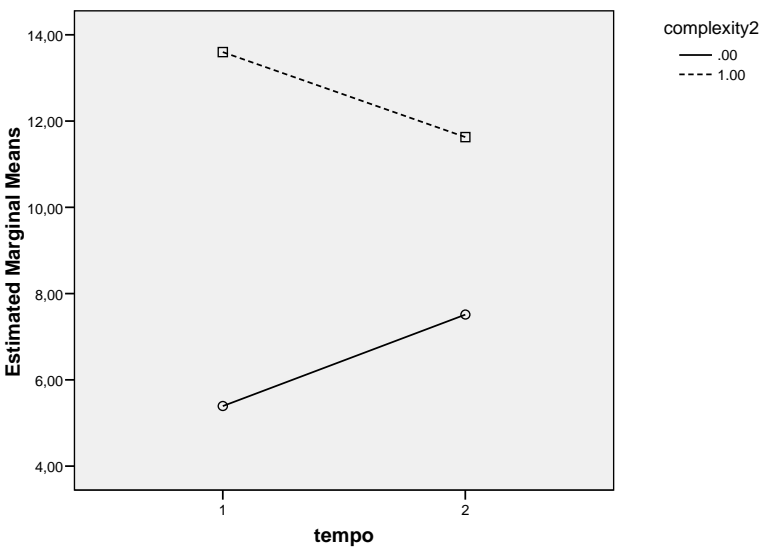

Legenda: $.00=$ menor complexidade; $1.00=$ maior complexidade; $1=1^{\mathrm{a}}$ aplicação; $2=2^{\mathrm{a}}$ aplicação

Figura 5 - Resultados da variável es nos grupos de menor e maior complexidade na $1^{\mathrm{a}}$ e $2^{\mathrm{a}}$ aplicações

6. Soma dos determinantes de sombreado difuso $\operatorname{Sum} Y[F Y+Y F+Y]$ - são as expressões de emoções vagas, de inquietação, ansiedade, e relacionam-se ao estresse situacional. Novamente observa-se uma confluência dos resultados. Após um ano de psicoterapia, o grupo de "menor" complexidade aumenta muito seus valores e no de "maior" complexidade, embora apresente uma diminuição, os valores se mantêm acima do esperado. Assim, observou-se o surgimento de emoções arcaicas, difusas e inquietantes nas pessoas mais superficiais, e naquelas com mais recursos essas emoções persistem, mas de uma forma menos intensa. Essa característica é esperada em um processo terapêutico, ou seja, a mobilização de aspectos emocionais paralisantes.

Estimated Marginal Means of MEASURE_1

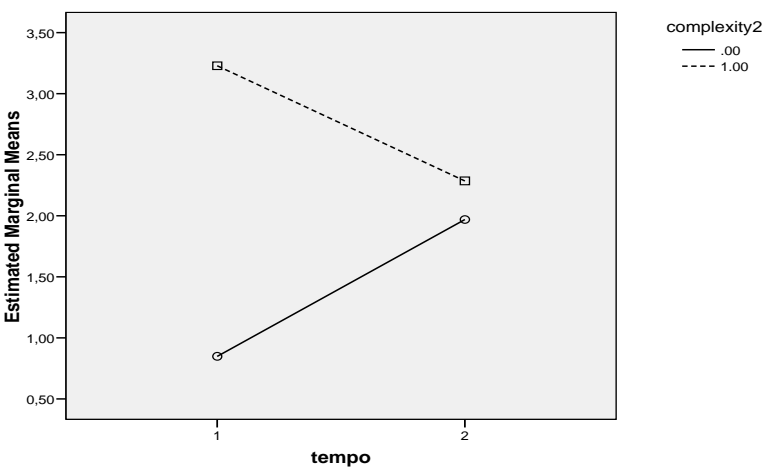

Legenda: $.00=$ menor complexidade; $1.00=$ maior complexidade; $1=1^{\mathrm{a}}$ aplicação; $2=2^{\mathrm{a}}$ aplicação

Figura 6 - Resultados da variável $\operatorname{Sum} Y$ nos grupos de menor e maior complexidade na $1^{\mathrm{a}}$ e $2^{\mathrm{a}}$ aplicações

7. Soma de todas as respostas de sombreado e cor acromática - SumShd [SumC'+Sum $T+\operatorname{Sum} Y+\operatorname{Sum} V]-$ refere-se à vivência de sobrecarga de estresse emocional. No grupo de "menor" complexidade, as pessoas mais superficiais de um valor abaixo do esperado passam para um valor próximo da expectativa na comparação entre os dois momentos.
Assim, de um funcionamento em que as emoções não se manifestavam, após um ano de terapia, passam a expressá-las. Já as pessoas de "maior" complexidade, de um valor elevado, revelando transbordamento das emoções e estresse elevado, apesar de passarem a um valor menor, ainda se mostram acima do esperado. Da mesma forma que as características anteriores, há um 
movimento de aproximação em ambos os estilos de funcionamento, com ambos os grupos permitindo a vivência de emoções dolorosas, que é um indicativo do processo psicoterápico.

Estimated Marginal Means of MEASURE_1

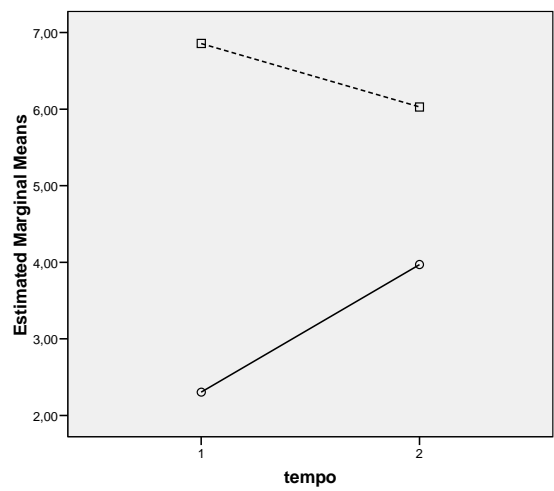

Legenda: $.00=$ menor complexidade; $1.00=$ maior complexidade; $1=1^{\mathrm{a}}$ aplicação; $2=2^{\mathrm{a}}$ aplicação

Figura 7 - Resultados da variável SumShd nos grupos de menor e maior complexidade na $1^{\mathrm{a}}$ e $2^{\mathrm{a}}$ aplicações

8. Determinantes Cor $-F C+C F+C+C n-$ trata-se da soma das respostas à cor que traduzem as reações e expressões afetivas. Na comparação entre os dois momentos nota-se um aumento das respostas associadas à cor no grupo de "menor" complexidade e uma sutil diminuição no de "maior" complexidade, embora permaneça acima do esperado. Observa-se, novamente, a confluência das tendências de ambos os grupos para valores mais próximos. As pessoas mais superficiais passaram a manifestar mais abertamente atitudes e respostas afetivas e as de mais recursos apontam uma moderação em sua expressão afetiva.

Estimated Marginal Means of MEASURE_1

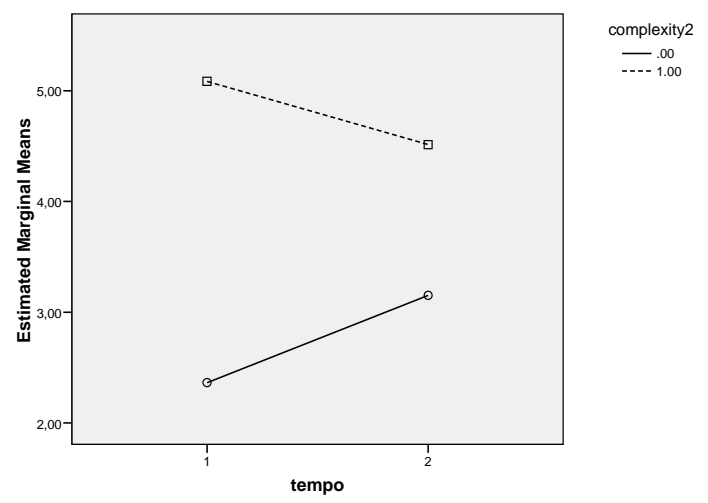

Legenda: $.00=$ menor complexidade; $1.00=$ maior complexidade; $1=1^{\mathrm{a}}$ aplicação; $2=2^{\mathrm{a}}$ aplicação

Figura 8 - Resultados da variável $F C+C F+C$ nos grupos de menor e maior complexidade na $1^{a}$ e $2^{a}$ aplicações

9. Soma dos determinantes Cor-forma e Cor pura $C F+C-$ diz respeito às reações afetivas mais imaturas, lábeis e impulsivas. Na representação gráfica, as linhas se cruzam na comparação entre os dois momentos. Isso porque tanto a elevação dos valores no grupo de "menor" complexidade quanto a queda no de "maior" complexidade foram acentuadas. Após um anos de psicoterapia, ambos os grupos apresentam valores acima do esperado. Assim, as reações mais impulsivas e os estados de humor oscilante, embora tenham diminuído nas pessoas de "maior" complexidade ainda se fazem presentes. Nas pessoas mais superficiais a terapia promoveu "maior" abertura e, portanto, surgem mais reações afetivas imaturas, e as de mais recursos foram adquirindo mais controle em seus comportamentos. 
Estimated Marginal Means of MEASURE_1

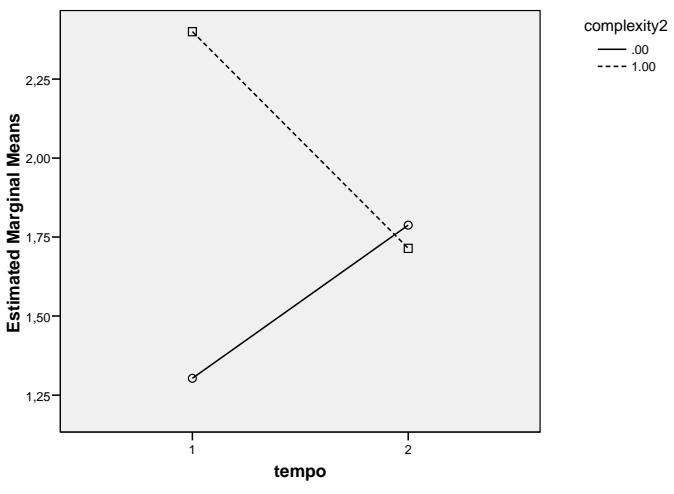

Legenda: $.00=$ menor complexidade; $1.00=$ maior complexidade; $1=1^{\mathrm{a}}$ aplicação; $2=2^{\mathrm{a}}$ aplicação

Figura 9 - Resultados da variável $C F+C$ nos grupos de menor e maior complexidade na $1^{\mathrm{a}}$ e $2^{\mathrm{a}}$ aplicações

10. Determinante de movimento humano $-M-$ refere-se ao uso da reflexão no comportamento, na solução de problemas, no planejamento do curso da ação deliberada. Relaciona-se também à capacidade para se identificar com as demais pessoas, portanto, à empatia e à noção da própria identidade. O grupo de "menor" complexidade apresentou aumento, aproximando-se daqueles esperados quando da comparação entre os dois momentos. Assim, após um ano de terapia, a abertura observada nas pessoa desse grupo é suficiente para autoafirmação, condições para enfrentar situações de desafio, pensar intencionalmente sobre a melhor forma de enfrentá-las. Já nas pessoas do grupo de "maior" complexidade, apesar da diminuição os valores, se mantiveram acima do esperado, o que associamos à diminuição da resposta motora das atuações. Ambos os grupos apresentam convergência dos resultados como nas variáveis anteriores.

Estimated Marginal Means of MEASURE_1

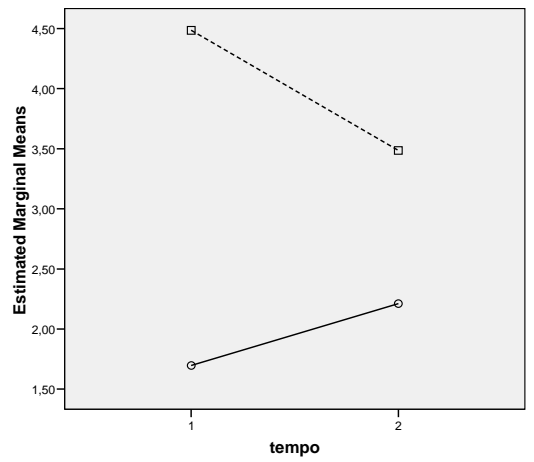

Legenda: $.00=$ menor complexidade $1.00=$ maior complexidade; $1=1^{\mathrm{a}}$ aplicação $2=2^{\mathrm{a}}$ aplicação

Figura 10 - Resultados da variável M nos grupos de menor e maior complexidade na $1^{\mathrm{a}}$ e $2^{\mathrm{a}}$ aplicações

12. Ativo - $a-$ ou movimento ativo, refere-se à flexibilidade de pensamento, perceber as experiências a partir de perspectivas diferentes e poder modificar pontos de vista, bem como abertura da mente para novas informações e idéias, flexibilidade ideacional nas tomadas de decisão. Observam-se diferenças entre os dois grupos e os gráficos de ambas comparações são semelhantes e mostram confluência dos resultados.
Em ambas comparações, as pessoas com protocolos de "menor" complexidade aumentaram sua tendência à flexibilidade mental em direção aos valores esperados, enquanto as mais complexas diminuem a excessiva abertura e flexibilidade para uma atitude de "maior" moderação, embora os valores finais ainda se mantenham um pouco acima do esperado. 
Estimated Marginal Means of MEASURE_1

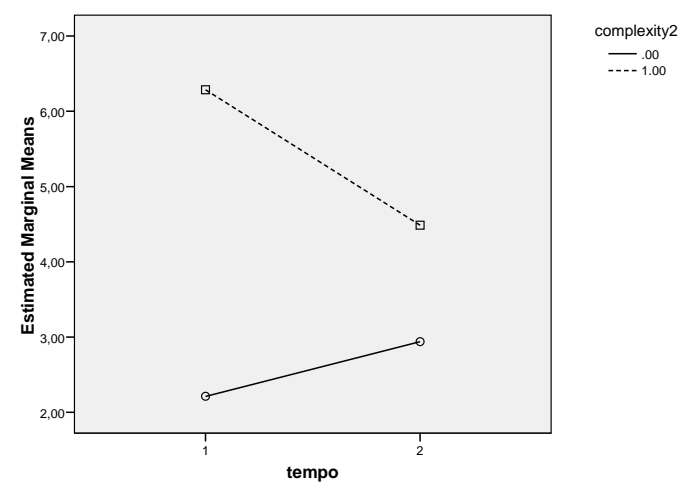

Legenda: $.00=$ menor complexidade; $1.00=$ maior complexidade; $1=1^{a}$ aplicação; $2=2^{a}$ aplicação

Figura 21 - Resultados da variável ativo nos grupos de menor e maior complexidade na $1^{\mathrm{a}}$ e $2^{\mathrm{a}}$ aplicações

13. Determinante de forma-dimensão - FD - refere-se à capacidade de introspecção suficiente que permite autoconsciência e autoinspeção adequadas. Nas comparações entre os momentos, as pessoas mais superficiais passaram de uma autoconsciência insuficiente para uma autoinspeção suficiente, com valores atingindo a expectativa média na segunda avaliação. Já as pessoas com mais recursos diminuíram seu investimento excessivo na introspecção, embora o mantenha em níveis um pouco acima do esperado. Como nas variáveis anteriores, há uma confluência dos resultados e, como consequência natural do processo terapêutico, observa-se o comportamento de olhar para si mesmo a partir de uma análise crítica, mas não negativa ou depreciativa.

Estimated Marginal Means of MEASURE_1

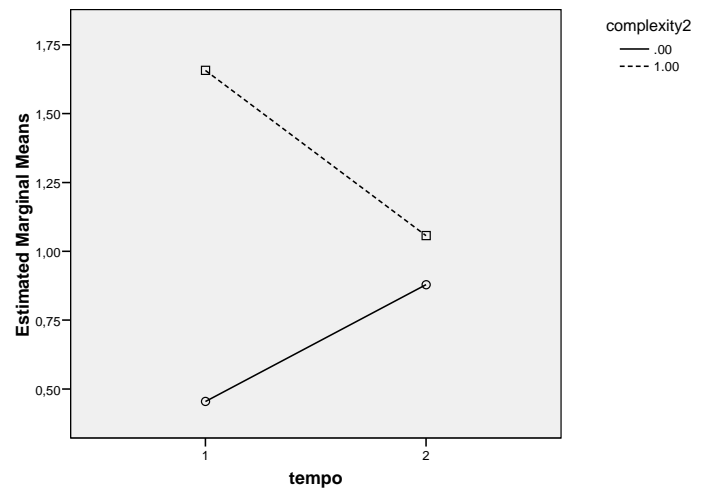

Legenda: $.00=$ menor complexidade; $1.00=$ maior complexidade; $1=1^{\mathrm{a}}$ aplicação; $2=2^{\mathrm{a}}$ aplicação

Figura 32 - Resultados da variável FD nos grupos de menor e maior complexidade na $1^{\mathrm{a}}$ e $2^{\mathrm{a}}$ aplicações

16. Soma de todas as respostas de conteúdo humano $H+(H)+H d+(H d)-$ inclui as imagens humanas totais e parciais, tanto reais quanto fantasiosas, e referem-se às noções que a pessoa tem se si mesma e dos outros e que participam do sentido da identidade. $\mathrm{Na}$ comparação entre os dois momentos, o grupo de "menor" complexidade manteve os valores iniciais próximos da média, enquanto o grupo de "maior" complexidade apresentou uma diminuição, mas que se mantém acima da expectativa. Assim, observa-se a manutenção do interesse por si e pelos outros. 
Estimated Marginal Means of MEASURE_1

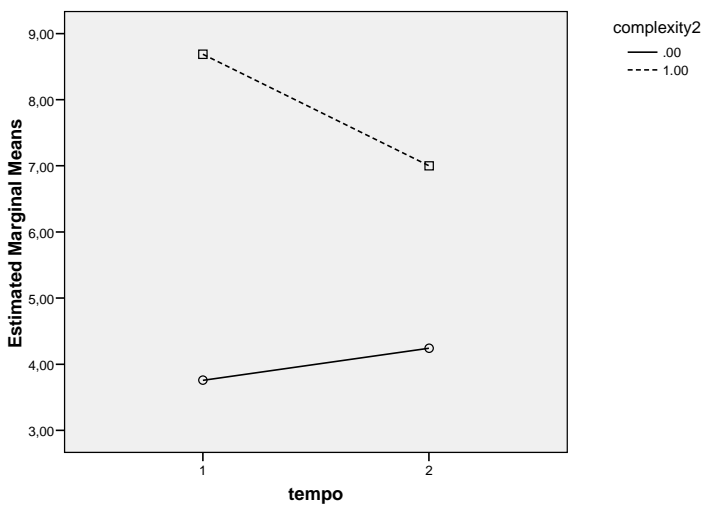

Legenda: $.00=$ menor complexidade; $1.00=$ maior complexidade; $1=1^{a}$ aplicação; $2=2^{a}$ aplicação

Figura 13 - Resultados da variável $H+(H)+H d+(H d)$ nos grupos de menor e maior complexidade na $1^{\mathrm{a}}$ e $2^{\mathrm{a}}$ aplicações

18. Respostas de conteúdo humano não puro - NPH refere-se às respostas de detalhe humano $[H d]$, de figura para humana $[(H)]$ e de detalhe de figura para humana $[(H d)]$, ou seja, presença de formas parciais da relação interpessoal ou identificação com figuras nãoreais, fantásticas, o que indica deficiências na identificação, desconforto social e dificuldades na adaptação nas relações com as demais pessoas. $\mathrm{Na}$ comparação entre os dois momentos ocorreu ligeiro aumento dessas imagens no grupo de "menor" complexidade, mas que se orienta em direção aos valores médios, enquanto se observa uma queda no grupo de "maior" complexidade mas que ainda se mantém acima da expectativa. Observa-se um movimento de liberdade de expressão das dificuldades relacionais nas pessoas mais contidas e melhora dessa manifestação naquelas de mais recursos pessoais.

Estimated Marginal Means of MEASURE_1

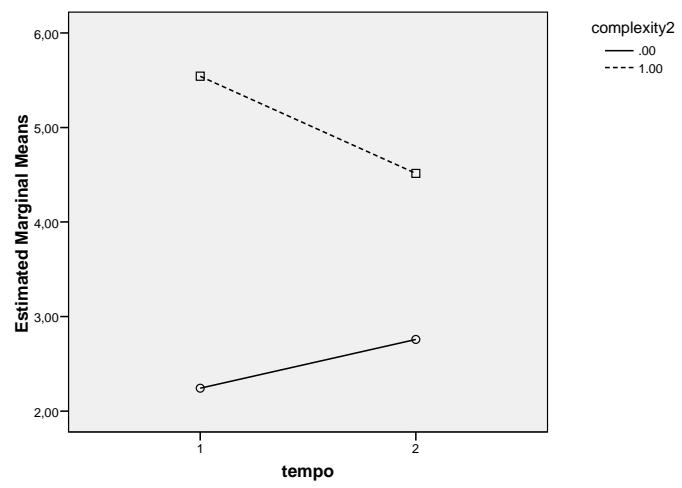

Legenda: $.00=$ menor complexidade; $1.00=$ maior complexidade; $1=1^{\mathrm{a}}$ aplicação; $2=2^{\mathrm{a}}$ aplicação

Figura 14 - Resultados da variável NHP nos grupos de menor e maior complexidade na $1^{\mathrm{a}}$ e $2^{\mathrm{a}}$ aplicações

19. Índice de depressão positivo_- DEPI - Composto por diversas variáveis relacionadas à autopercepção negativa, denegrida ou grandiosa, à presença de sentimentos de ambivalência, de atitude de oposição e negativismo, de menor sensibilidade afetiva ou responsividade superficial, presença de componentes emocionais superando os de reflexão, presença de racionalização, reduzida atitude afetiva de aproximação. Essas variáveis indicam a presença dos diferentes aspectos da personalidade envolvidos no funcionamento depressivo, seja no estado de sobrecarga emocional e disforia acompanhando a manifestação afetiva, seja na esfera cognitiva, referente à distorção da autopercepção. O grupo de "maior" complexidade apresentou uma queda na pontuação positiva de depressão após um ano de terapia, 
enquanto o grupo de "menor"complexidade elevou seus valores. Isso significa que de uma menor sensibilidade emocional e afetiva dada ao retraimento, esse grupo de “menor"complexidade passa a expressar desconforto emocional, o que, do ponto de vista do processo psicoterapêtico, constitui um fator de desenvolvimento pessoal.

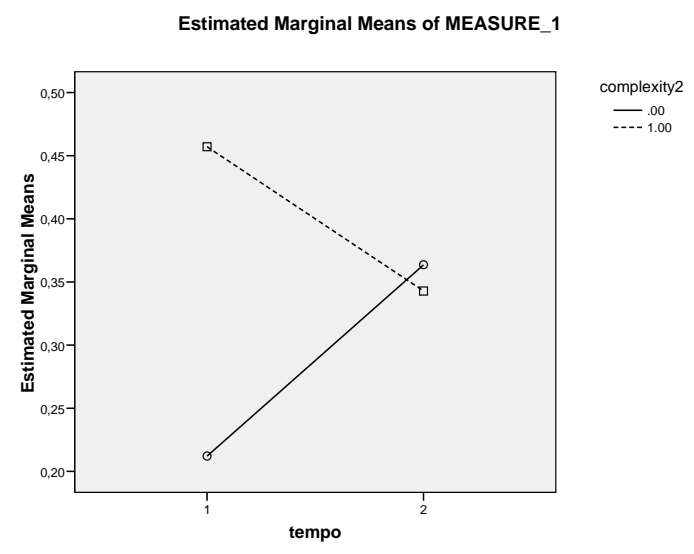

Legenda: $.00=$ menor complexidade; $1.00=$ maior complexidade; $1=1^{\mathrm{a}}$ aplicação; $2=2^{\mathrm{a}}$ aplicação

Figura 45 - Resultados da variável DEPI pos. nos grupos de menor e maior complexidade na $1^{\mathrm{a}}$ e $2^{\mathrm{a}}$ aplicações

\section{Conclusão}

Esses achados permitem várias considerações. Foi interessante constatar resultados em uma série de variáveis dos grupos de "menor" e "maior" complexidade que confluíram em direção a valores mais próximos das expectativas no decorrer do processo psicoterápico.

Dessa forma, ocorreram mudanças em direção à maior produção associativa, maior abertura para a experiência e manifestação de recursos internos nas pessoas inicialmente mais inibidas. Por outro lado, as pessoas mais complexas passam para uma atitude de menor exposição e maior contenção ou filtro das expressões internas. Assim, a psicoterapia permitiu o "maior" aproveitamento do potencial interno, ideativo e afetivo dessas pessoas.

As pessoas que inicialmente apresentaram menos recursos internos conseguiram trazer à tona suas ansiedades e angústias, mas agora em um contexto inter-relacional, o que permite a vivência e a elaboração desses aspectos. Por outro lado, nas pessoas mais complexas, os aspectos ideativos intrusivos e fantasiosos, e as inquietações, o nervosismo e as emoções disfóricas passam a se expressar com menor vigor. Faz parte do processo terapêutico a mobilização dos aspectos afetivos, emocionais e ideativos e, no presente estudo, a terapia de longa duração permitiu que as pessoas cada vez mais expressassem seu desconforto emocional, suas angústias, suas ansiedades, seus medos e passassem a mostrar as vivências internas dolorosas. É a mobilização das vivências subjetivas que possibilita sua elaboração durante o processo psicoterápico.

Da mesma forma, a psicoterapia permitiu o surgimento e o aumento da tendência à aproximação emocional com o outro, ao contato interpessoal caracterizado pela maior confiança nas pessoas, nas relações interpessoais, promovido pelo processo psicoterápico. Esse aumento da confiança nos outros leva as pessoas atendidas a estabelecerem vínculos mais íntimos nas relações interpessoais. Elas também passaram a se interessar mais pelos outros e por si mesmas, deixaram de apresentar tendências ao isolamento e de buscar proteção ou conforto em ambientes mais simples e conhecidos, uma vez que mostram ter mais condições de enfrentar as demandas de ambientes mais competitivos e complexos.

Outra melhora decorrente da psicoterapia diz respeito à percepção de si e do outro. As pessoas com protocolos de "maior" complexidade diminuíram a tendência inicial de compensar o sentimento de inferioridade por meio da tendência à grandiosidade, ao exagero do valor pessoal. Já as pessoas inicialmente menos complexas aumentaram sua autopercepção, autoconsciência e autoestima. Tanto as pessoas menos quanto as mais complexas passam a se perceber mais, a ter uma concepção mais realística de si e dos outros, a ter uma melhor noção da própria identidade baseada em valores pessoais, o que promove a empatia com os outros.

Quanto às reações e respostas afetivas, ambos os grupos, de "maior" e "menor" complexidade, convergem em direção às mais amadurecidas, com 
maior consideração pela realidade, maior adequação, controle e modulação na resposta afetiva. Entretanto, no que se refere às respostas afetivas imaturas, estas diminuem no grupo de "maior" complexidade e aumentam no de "menor" complexidade. Assim, as pessoas mais inibidas passam a manifestar reações afetivas mais lábeis, enquanto as com mais recursos passam a conter as manifestações afetivas instáveis e impulsivas. Faz sentido esse resultado, pois as pessoas mais contidas se permitem, após um ano de terapia, expor suas tendências mais reativas e menos controladas, o que é positivo. Já aquelas que se expõem mais passam a refrear as tendências imediatas e reativas que se faziam presentes.

$\mathrm{O}$ aumento da flexibilidade de pensamento nas pessoas com menos recursos mostrou que passaram a conseguir observar os fatos sob diferentes pontos de vista. Por outro lado, a redução dessa possibilidade nas pessoas com mais recursos não indica aumento da rigidez, mas, sim, a diminuição da oscilação entre diferentes perspectivas em direção a uma estabilização como consequência da psicoterapia.

As dificuldades de manejo e controle das situações de estresse diminuem ao longo dos dois anos em ambos os grupos, de "menor" e "maior" complexidade. Dessa forma, todas as pessoas atendidas passaram a conseguir fazer uso de recursos pessoais apropriados que lhes permitem manejo adequado das demandas externas e internas. Desse modo, ocorre também uma nítida melhora no lidar com as circunstâncias cotidianas, com as experiências de vida e com as exigências dos mundos interno e externo.

Por tudo que foi relatado, pode-se dizer que a psicoterapia psicanalítica realizada em ambulatório universitário gratuito, em sua maioria por profissionais em treinamento, promoveu mudanças psíquicas importantes nas pessoas que foram assistidas.

\section{Referências}

American Psychiatric Association (1994). Diagnostic and statistical manual of mental disorders ( $4^{\mathrm{a}} \mathrm{Ed}$.). Washington, DC: Author.
Del-Ben, C. M., Vilela, J. A. A., Crippa, J. A. S., Hallak, J. E. C., Labate, C. M. \& Zuardi, A. W. (2001). Confiabilidade da "Entrevista Clínica Estruturada para o DSM-IV - Versão Clínica" traduzida para o português. Official Journal of the Brazilian Psychiatric Association, 23(3), 156-159.

Dean, K. L., Viglioni, D. J., Perry, W. \& Meyer, G. J. (2007). A method to optimize the response range while maintaining Rorschach comprehensive system validity. Journal of Personality Assessment, 89(2), 149-161.

Exner, J. E., Jr. (2003). The Rorschach: a comprehensive system, Vol 1. Basic foundations and principles of interpretation (4 ${ }^{\mathrm{a}}$ Ed.). New Jersey: John Wiley \& Sons.

Morse J. M. \& Field, P. A. (1995). Qualitative research method for health professionals ( $2^{\mathrm{a}} \mathrm{Ed}$.). Londres: Sage Publications.

Patton, M. Q. (2002). Qualitative research \& evaluation methods ( $3^{\mathrm{a}}$ Ed.). Londres: Sage Publications.

Primi, R., Munhoz, A. M. H., Bighetti, C. A., Nucci, E. P., Pelegrini, M. C. K. \& Moggi, M. A. (2000). Desenvolvimento de um inventário de levantamento das dificuldades da decisão profissional. Psicologia: Reflexão e Crítica, 13(3), 451463.

Tabachinick, B. G. \& Fidell, L. S. (1996). Using multivariate statistics. Nova Iorque: Harper Collins.

Turato, E. R. (2003). Tratado da metodologia da pesquisa clinico-qualitativa. Petrópolis, RJ: Vozes.

Weiner, I. B. (2000). Princípios da interpretação do Rorschach (M. C. V. M. Silva, trad.). São Paulo: Casa do Psicólogo. 
Sobre as autoras:

Latife Yazigi é psicóloga com graduação e pós-graduação na Universidade de São Paulo, pós-doutorado na Universidade de Chicago, livre-docente e professora titular da Universidade Federal de São Paulo, bolsista 1B do CNPq e vice-presidente da International Rorschach Society. É Membro do Comitê de Ciências Humanas, Psicologia, da Fundação de Amparo à Pesquisa do Estado de São Paulo.

Terezinha de Carvalho Amaro é psicóloga, doutora em Ciências com Pós-Doutorado pela Universidade Federal de São Paulo, apoio FAPESP. Especialista em Psicologia Clínica e Hospitalar pelo Conselho Regional de Psicologia, é membro de diretoria da Associação Brasileira de Psicossomática, Regional São Paulo e professora do Curso de Psicologia da Universidade Presbiteriana Mackenzie nas áreas de Avaliação Psicológica e Pesquisa.

Maria Luiza de Mattos Fiore é psicanalista da Sociedade Brasileira de Psicanálise - Associação Psicanalítica Internacional - IPA- (2005); doutora pelo Departamento de Psiquiatria, Universidade Federal de São Paulo (1997); mestre pelo Departamento de Psiquiatria, Universidade Federal de São Paulo, (1992); médica pela Universidade Federal de São Paulo (1982) e pesquisadora do Centro Clínico de Pesquisa em Psicoterapia da Universidade Federal de São Paulo.

Norma Lottenberg Semer é psicóloga pela USP e doutora em Saúde Mental pela UNIFESP, onde desenvolve funções de assistência, docência e pesquisa na Disciplina de Psicoterapia do Departamento de Psiquiatria. É vicepresidente da Associação Brasileira de Rorschach de São Paulo e psicanalista membro associada da Sociedade Brasileira de Psicanálise de São Paulo.

Apoio FAPESP, CNPq. 